\title{
A vector-based short hairpin RNA targeting Aurora A inhibits breast cancer growth
}

\author{
YANG WAN $^{1}$, ANLIANG HUANG ${ }^{1}$, YANG YANG ${ }^{1}$, GANG XIE $^{1}$, XIANG CHEN $^{1}$, JIA HU ${ }^{1}$, XIANCHENG CHEN $^{1}$, \\ LI YANG $^{1}$, JIONG LI ${ }^{1}$, LIJUAN CHEN ${ }^{1}$, YU JIANG ${ }^{1}$, XIA ZHAO $^{2}$, YUQUAN WEI ${ }^{1}$ and HONGXIN DENG ${ }^{1}$ \\ ${ }^{1}$ State Key Laboratory of Biotherapy, West China Hospital and West China Medical School, Sichuan University; \\ ${ }^{2}$ Department of Gynecology and Obstetrics, West China Second Hospital, Sichuan University, \\ Ke-yuan Road 4, No. 1, Gao-peng Street, Chengdu, Sichuan, 610041, P.R. China
}

Received November 26, 2009; Accepted January 25, 2010

DOI: 10.3892/ijo_00000594

\begin{abstract}
Aurora A plays an essential role in centrosome maturation, separation and in the formation of the mitotic bipolar spindle. Overexpression or amplication of Aurora A gene has been detected in many cancer cell lines and various tumor tissues, including breast cancer, suggesting that Aurora A might be drug target for breast cancer treatment. In the current study, short hairpin RNA targeting Aurora A was cloned into pGenesil-2 plasmid vector and then transfected into MDA-MB-435S and ZR-75-30 human breast cancer cells using cationic liposome. Reduced expression of Aurora A was detected by RT-PCR and Western blot. The effect of pGenesil-2-shAURKA plasmid on tumor growth in MDAMB-435S xenogenic implantation model was studied. pGenesil-2-shAURKA plasmid inhibited tumor growth significantly by systemantic administration. To further study the underlying mechanisms, cell apoptosis and proliferation were investigated by flow cytometric analysis, propidium iodide staining, TUNEL and $\mathrm{Ki}-67$ immunostaining respectively. Increased apoptosis and reduced cell proliferation were detected in vitro and in vivo studies. In summary, our results suggested that specific knockdown of Aurora A expression by vector based shRNA may be a potential therapy for human breast cancer.
\end{abstract}

\section{Introduction}

Breast cancer is the most commonly diagnosed cancer in women worldwide. More than 1.15 million women were diagnosed with breast cancer in 2002 (1). Registry data showed that breast cancer incidence has been increasing since 1973, especially in low and middle-income countries.

Correspondence to: Dr Hongxin Deng, State Key Laboratory of Biotherapy, West China Hospital and West China Medical School, Sichuan University, Ke-yuan Road 4, No. 1, Gao-peng Street, Chengdu, Sichuan, 610041, P.R. China

E-mail: denghongx@scu.edu.cn

Key words: Aurora A, short hairpin RNA, breast cancer
In China, urban registries showed $20-30 \%$ increase in the past decade (2). Current breast cancer incidence and mortality rates $(\sim 180,510$ and 40,940 cases in 2007 in the United States, respectively) (3) highlight the need to explore alternative therapeutic strategies.

The process of cell division is instrumental to the development and progression of tumors, and targeting cell division has been proved as a successful antitumor therapy. A number of trials has addressed the benefit of adding a taxane (paclitaxel or docetaxel) to an anthracycline-based adjuvant chemotherapy regimen in breast cancer $(4,5)$, suggesting that the molecules involved in cell cycle and division may be targets for cancer treatment.

Aurora $\mathrm{A}$ is an oncogenic serine/threonine kinase that plays an essential role in centrosome maturation, separation and in the formation of the mitotic bipolar spindle in various organisms (6-10). It has been reported that its ectopic expression in immortalized NIH/3T3 cells is sufficient to provoke their transformation, defining Aurora A as an oncogene (11). Overexpression or amplication of Aurora A gene has been detected in many cancer cell lines and various tumor tissues, including breast cancer (reviewed in ref. 12). Nadler et al reported that Aurora A expression defines a population of patients with decreased survival, whereas Aurora B expression does not, suggesting that Aurora A might be the preferred drug target in breast cancer (13).

RNA interference is a specific way of gene silencing and a plethora of in vitro and in vivo proof-of-concept exist. It has been shown that practically many human diseases with a gain-of-function genetic lesion can become a target for therapeutic RNAi, including cancer, viral infections, neurodegenerative diseases and ocular disorders (14-16). Short hairpin RNAs (shRNAs) with a stem-loop 'hairpin' structure driven by polymerase III promoters have been investigated as an alternative strategy to suppress gene expression more stably, and such constructs with well-defined initiation and termination sites have been used to produce various small dsRNA species that inhibit the expression of genes with diverse functions in mammalian cell lines (17). In the current study, we recombined an shRNA targeting Aurora A into a plasmid vector and studied the effects on expression of Aurora A and tumor growth in vitro and in vivo. 
Table I. Primer sequences for Aurora A and B, and B-actin.

\begin{tabular}{llll}
\hline Gene & & Primers' sequences & Products size (bp) \\
\hline Aurora A & Forward: & GAGGCAGTGGGCTTTGG & 515 \\
& Reverse: & GGCAGGTAGTCCAGGGTG & 576 \\
Aurora B & Forward: & ATCTTAACGCGGCACTTCAC & \\
& Reverse: & GCACCACAGATCCACCTTCT & 434 \\
B-actin & Forward: & CGGGAAATCGTGCGTGAC & \\
& Reverse: & TGGAAGGTGGACAGCGAGG & \\
\hline
\end{tabular}

\section{Materials and methods}

Cell lines. Human breast cancer cell lines, MDA-MB-435S and ZR-75-30, were obtained from the American Type Culture Collection (Manassas, VA, USA). The two cell lines were grown in Dulbecco's modified Eagle's medium supplemented with $10 \%$ FBS (Gibco, Carlsbad, CA, USA), in a $37^{\circ} \mathrm{C}$-incubator with a humidified $5 \% \mathrm{CO}_{2}$ atmosphere.

Plasmid constructions. pGenesil-2 vectors harboring caccgATGCCCTGTCTTACTGTCAttcaagagaTGACAGTA AGACAGGGCATtttttg (18) or caccgCGTACGCGGAA TACTTCGAttcaagagaTCGAAGTATTCCGCGTACGtttttg at its BpiI/BamHI sites were prepared for expressing short hairpin RNAs (shRNAs), specific for interfering with expressions of Aurora A (pGenesil-2-shAURKA) or as irrespective sequence control (pGenesil-2-shHK), respectively. The capital charaters refer to the specific sequece for Aurora A or for control, and the italic characters refer to the loop structure of shRNA. All the constructed plasmids were confirmed by DNA sequencing. Colonies of Escherichia coli containing pGenesil-2-shAURKA or pGenesil-2-shHK were cultured in LB broth containing $50 \mu \mathrm{g} / \mathrm{ml}$ of kanamycin. Large-scale plasmid DNA was purified using an EndoFree Plasmid Giga kit (Qiagen, Hilden, Germany). The DNA was eventually dissolved in sterile endotoxin-free water and stored at $-20^{\circ} \mathrm{C}$ before use.

Liposome preparation. A cationic liposome for plasmid transfection and treatment in animal experiments was used (DOTAP/cholesterol). The cationic liposome was prepared using the procedure described previously (19). Briefly, a lipid film was prepared by rotary evaporation at $30^{\circ} \mathrm{C}$ for 30 min from a mixture of DOTAP and cholesterol (Avanti Polar Lipids, Alabaster, AL, USA) at 1:1 molar ratio in chloroform. The film was rehydrated in $5 \%$ dextrose, vortexed for $30 \mathrm{~min}$ at $60^{\circ} \mathrm{C}$. Then, the mixture was left overnight and sonicated at low frequency for $5 \mathrm{~min}$ at $50^{\circ} \mathrm{C}$. After sonication, it was transferred to a tube and heated at $50^{\circ} \mathrm{C}$ for $10 \mathrm{~min}$. Then it was extruded through a $100 \mathrm{~nm}$ polycarbonate filter by using an Avanti Polar Lipids MiniExtruder. The final cationic liposome (DOTAP/cholesterol) was a small multi-lamellar liposome in a size range of $100 \pm 20 \mathrm{~nm}$. It was stored under argon gas at $4^{\circ} \mathrm{C}$.

Transfection. Twenty-four hours before transfection, cells were trypsinized and seeded in 6 -well culture plates at $1 \times 10^{5}$ cells per well. DNA (pGenesil-2-shAURKA or pGenesil-2-shHK)/ liposome complexes were prepared in DMEM medium, which contained $2 \mu \mathrm{g}$ DNA and $5 \mu \mathrm{g}$ liposome, and left at room temperature for $30 \mathrm{~min}$. In addition, $5 \mu \mathrm{g}$ liposome or medium alone were also used as control. The cells were incubated with the above agents for $4 \mathrm{~h}$, rinsed three times with PBS and then $1.5 \mathrm{ml}$ of DMEM supplemented with $10 \%$ FBS was added to each well, with a continued incubation for an additional $48 \mathrm{~h}$ for further studies.

RNA extraction and RT-PCR. Total RNA from cells or tumor tissues were extracted using TRIzol (Invitrogen, Carlsbad, CA, USA) as recommended by the manufacturer. Reverse transcription-polymerase chain reaction (RT-PCR) was used for the analysis of Aurora A, Aurora B mRNA with B-actin as an internal control. RNA $(1 \mu \mathrm{g})$ was reverse transcribed using SuperScript II reverse transcriptase (Invitrogen) and oligo(dt) primer. Primers for Aurora A, Aurora B and Bactin are shown in Table I. PCR was performed by initial denaturation at $94^{\circ} \mathrm{C}$ for $2 \mathrm{~min}$ followed by 30 cycles of $30 \mathrm{sec}$ at $94^{\circ} \mathrm{C}, 30 \mathrm{sec}$ at $58^{\circ} \mathrm{C}$ and $60 \mathrm{sec}$ at $72^{\circ} \mathrm{C}$. All PCR products were separated by electrophoresis in $1 \%$ agarose gels and visualized using ethidium bromide. Experiments were performed in triplicate.

Western blot. Cells were lysed on ice for 30 min with RIPA Lysis Buffer (containing 50 mM Tris-HCl, pH 7.4; 1\% NP-40; $0.25 \%$ Na-deoxycholate; $150 \mathrm{mM} \mathrm{NaCl} ; 1 \mathrm{mM}$ EDTA; $1 \mathrm{mM}$ PMSF; $1 \mu \mathrm{g} / \mathrm{ml}$ aprotinin, leupeptin, pepstatin each; $1 \mathrm{mM}$ $\mathrm{Na}_{3} \mathrm{VO}_{4} ; 1 \mathrm{mM} \mathrm{NaF}$ ). For tissue samples, a frozen piece of tissue was powdered and lysed in RIPA lysis buffer. The proteins $(10 \mu \mathrm{g})$ were separated by SDS-polyacrylamide gel electrophoresis (SDS-PAGE) and electronically transferred onto a polyvinylidene difluoride membrane (Millipore, Bedford, MA). After blocking, the membranes were incubated with recommended dilution primary antibodies against Aurora A, Aurora B or B-actin (Abcam, Cambridge, MA, USA), followed by incubation with peroxidase-conjugated secondary antibodies (Abcam). Peroxidase-labeled bands were visualized using an ECL kit (Pierce, Rockford, IL, USA). The ratio of Aurora A (or Aurora B)/B-actin was calculated by using densitometry, and values were normalized by dividing by the ratio at no treatment.

Flow cytometric analysis. Flow cytometric analysis (FCM) was carried out to identify sub-G1 cells. Cells were harvested with trypsin-EDTA, washed with PBS, and pelleted and 
suspended in PI/RNase/PBS (100 $\mu \mathrm{g} / \mathrm{ml}$ propidium iodide and $10 \mu \mathrm{g} / \mathrm{ml}$ RNase A). Analysis of DNA content was done on a FACSCalibur system (BD Immunocytometry Systems, San Jose, CA, USA).

Nuclear staining and fluorescent microscopy. Morphological evaluation of the nuclei of untreated and treated cells was determined by nuclear staining with propidium iodide (PI). Briefly, treated and untreated cells in 6-well culture plates were washed twice with PBS and fixed in $4 \%$ formaldehyde in PBS for $20 \mathrm{~min}$ at room temperature, rinsed 3 times with PBS and permeabilized with methanol at $-20^{\circ} \mathrm{C}$ for $10 \mathrm{~min}$. Fixed cells were stained with $20 \mu \mathrm{g} / \mathrm{ml} \mathrm{PI}$, and subsequently examined using a Zeiss Axiovert 400 microscope and Axio Cam MRm camera (Carl Zeiss, München-Hallbergmoos, Germany).

Animal studies. All animal procedures were approved by the Institutional Animal Care and Treatment Committee of Sichuan University. MDA-MB-435S mouse tumor model was established by injecting $2 \times 10^{6}$ MDA-MB-435S cells subcu-taneouly into the flanks of 6-8 weeks old female athymic nude mice on day 0 . When tumors were palpable 10 days later, mice $(\mathrm{N}=5)$ were injected intravenously through the tail vein with $100 \mu 1$ of liposomal complexes containing $5 \mu \mathrm{g}$ of the pGenesil-2-shAURKA or pGenesil-2-HK plasmid and $15 \mu \mathrm{g}$ liposome or $100 \mu 15 \%$ glucose as control 3 times a week (on Monday, Wednesday, Friday) for 4 weeks. Tumors were measured twice a week with vernier calipers. Tumor volume was calculated according to the following formula: volume $=0.52 \mathrm{x}(\text { width })^{2} \mathrm{x}$ length . Animals were sacrificed 3 days after the last treatment, and tumors were excised and weighed. Part of the tumor tissue was immediately frozen and the rest was fixed in $10 \%$ neutral-buffered formalin and embedded in paraffin. To monitor drug toxicity, body weight were measured and a pathologist examined the mouse organs, including heart, liver, spleen, lungs and kidneys.

Histologic studies. Breast tumor, heart, liver, spleen, lungs and kidneys were removed, fixed in neutral-buffered formalin and embedded in paraffin. Sections ( $3 \mu \mathrm{m}$ thick) were cut and stained with $\mathrm{H} \& \mathrm{E}$ for histologic evaluation.

Immunohistochemistry. The rabbit anti-Aurora A or Ki-67 antibodies and dilutions were used for immunohistochemistry. After deparaffinization, antigen retrieval was performed by heating slides in autoclave in $10 \mathrm{mM}$ sodium citrate buffer at pH 6.0 for 5 min after pressure gainning, and sections were incubated with the rabbit Aurora A or Ki-67 antibodies at $4^{\circ} \mathrm{C}$ overnight. As the second step, biotinylated goat antirabbit IgG was applied and detected by using of the SABC Elite kit (Boster, Wuhan, China) with diaminobenzidine as substrate. For the quantitative comparison, Ki-67-labeling index was calculated as the percentage of neoplastic cells with positive nuclear staining in the total number of neoplastic cells.

In situ terminal deoxynucleotidyl transferase mediated dUTP nick end-labeling assay. Terminal deoxynucleotidyl transferase-mediated dUTP nick-end labeling (TUNEL) staining was performed with an in situ apoptotic cell detection kit according to the manufacturer's directions (Promega, Madison, WI, USA). Briefly, tumor sections were treated with a $20 \mu \mathrm{g} / \mathrm{ml}$ proteinase $\mathrm{K}$ solution and incubated with rTdT incubation buffer. Then the images of the representative sections were taken by using the microscope and Axio Cam MRm camera. Five equal-sized fields were randomly chosen and analyzed. Density was evaluated in each field to calculate the density of apoptotic cells (apoptosis index).

Statistical analysis. All data are presented as means \pm SD. Data were analyzed by one-way ANOVA and Tukey's test. Differences between means as appropriate were considered significant when yielding $\mathrm{P}<0.05$. Experiments were performed at least in duplicate.

\section{Results}

Specific knockdown of Aurora A in breast cancer cell lines. The pGenesil-2-shAURKA plasmid was transfected into two breast cancer cells, MDA-MB-435S and ZR-75-30, respectively. After $48 \mathrm{~h}$, the cells were harvested, and their expression levels of Aurora A were analyzed by RT-PCR and Western blot. As shown in Fig. 1, in both mRNA and protein levels, dramatic suppression of Aurora A expression was observed in the two cell lines treated with pGenesil-2-shAURKA. The pGenesil-2-shAURKA plasmid transfection did not cause a non-specific inhibition of gene expression, as shown by expres-sions of Aurora B. Quantification of band intensities of Aurora A in the Western blot showed that the treatment with pGenesil-2-shAURKA plasmid reduced the expression of Aurora A by $63 \%$ in MDA-MB-435S cells and $72 \%$ in ZR-75-30 cells $(\mathrm{P}<0.001)$.

Knockdown of Aurora A induces cell apoptosis in vitro. The quantitative assessment of sub-G1 cells by FCM was used to estimate the number of apoptotic cells. As shown in Fig. 2A, an increase in the sub-G1 phase population was observed after pGenesil-2-shAURKA treatment in MDA-MB-435S and ZR-75-30 cell lines by flow cytometric analysis $(\mathrm{P}=0.002$ and $\mathrm{P}=0.006$, respectively). To further assess apoptosis by morphological changes, nuclear staining with PI was performed. As shown in Fig. 2B, the morphological changes of cells at $48 \mathrm{~h}$ post-transfection monitored by fluorescence microscopy were characteristic of apoptosis (rounded or floating). These results indicated that the shRNA-mediated knockdown of Aurora A led the breast cancer cells to apoptosis.

Knockdown of Aurora A inhibited the growth of MDA-MB$435 \mathrm{~S}$ cells in vivo. We further examined whether pGenesil-2shAURKA could inhibit the growth of MDA-MB-435S cells in vivo. As shown in Fig. 3A, from day 24 after tumor implantation, the tumor volume in pGenesil-2-shAURKAtreated mice started to show significant differences from those in controls $(\mathrm{P}<0.05)$. To evaluate the tumor growth more accurately, animals were sacrificed on day 42 after tumor implantation, and tumors were excised and weighed. Tumors of mice treated with glucose and pGenesil-2-HK 
A

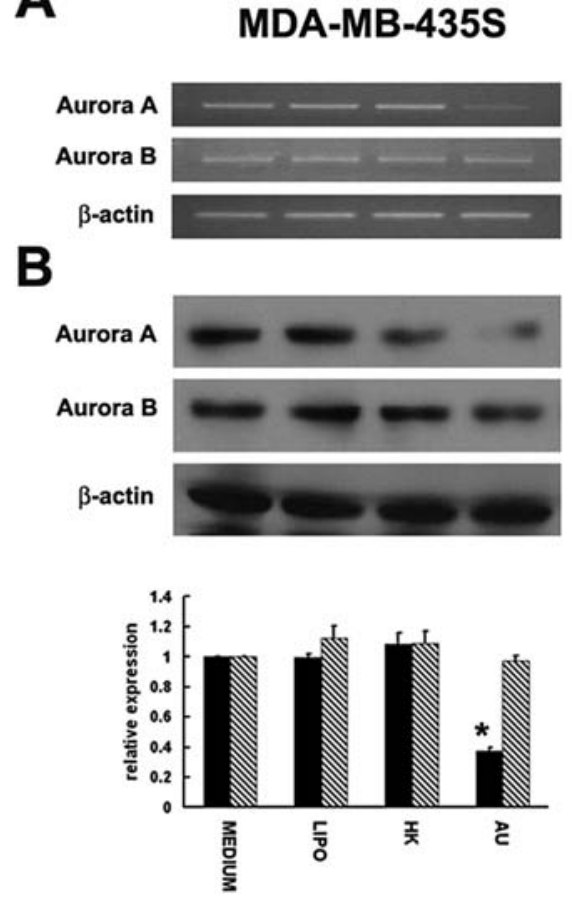

ZR-75-30
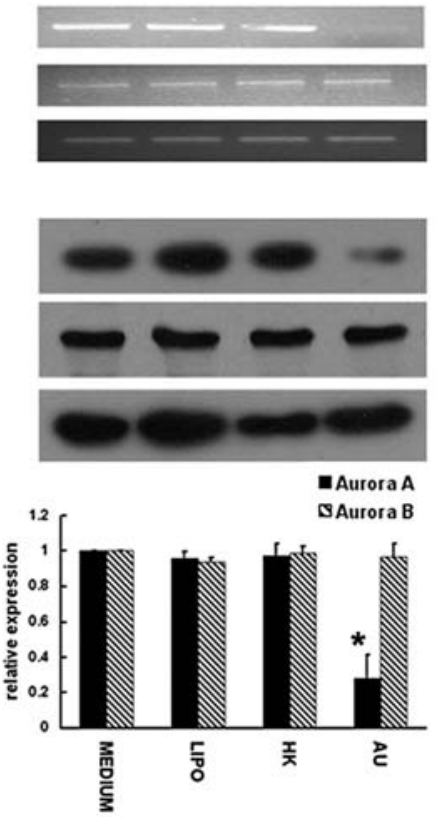

Figure 1. pGenesil-2-shAURKA specifically inhibits Aurora A expression. Human breast cancer cell lines, MDA-MB-435S and ZR-75-30 were transfected with pGenesil-2-shAURKA (AU) or pGenesil-2-shHK (HK)/liposome complex or liposome (LIPO) or medium (MEDIUM) alone as control for 48 h. (A) Expression of Aurora A and B were detected by RT-PCR. (B) Upper panel, expression of Aurora A and B were detected by Western blot, B-actin expression was monitored as the control. The ratio of AURKA (or AURKB)/ $\beta$-actin was calculated by using densitometry, and values were normalized by dividing by the ratio at MEDIUM group. The experiments were performed 3 times and the average ratio of Aurora A (or Aurora B)/ß-actin are shown in lower panel. Values are means $\pm \mathrm{SD} .{ }^{*} \mathrm{P}<0.05$ compared with MEDIUM group.

plasmid reached $1.632 \pm 0.731 \mathrm{~g}$ and $1.138 \pm 0.819 \mathrm{~g}$ in weight, respectively. However, tumors in mice treated with pGenesil2-shAURKA were significantly smaller (Fig. 3B, P<0.001), reaching only $0.028 \pm 0.013 \mathrm{~g}$ in weight. Histopathological analysis of control mice showed a typical hypercellular solid carcinoma invading the dermis and subcutaneum. In contrast, treated mice showed a marked reduction in tumor volume, partial encapsulation by fibrous connective tissue, and no significant invasion into surrounding skin tissue (Fig. 3C).

We further examined the expression of Aurora A and B in tumor tissues. Similar to the results in vitro, systematic administration of pGenesil-2-shAURKA plasmid reduced the expression of Aurora A by $72 \%(\mathrm{P}<0.001)$ and had little effect on Aurora B expression (Fig. 4).

In addition, the mice treated with pGenesil-2-shAURKA had been investigated for potential side-effects. No significant adverse consequences were observed in gross measures, including weight loss, ruffling of fur, life span, behavior and feeding. Furthermore, no pathological changes in heart, liver, spleen, lungs, or kidneys were found by microscopic examination (data not shown).

Knockdown of Aurora A induces cell apoptosis in vivo. TUNEL assays were carried out to detect apoptosis in tumor tissues. As shown in Fig 5A, within a similar field of view, more apoptotic cells were observed in tumor tissues from the mice treated with pGenesil-2-shAURKA plasmid than glucose and pGenesil-2-HK treatment groups. For quantitative comparison, the apoptosis index in each group was calculated. The apoptosis index was significantly higher in pGenesil-2-shAURKA treatment group than glucose and pGenesil-2-HK treatment groups with values of $6.86 \pm 1.32 \%$ vs. $2.82 \pm 0.42 \%$ and $1.67 \pm 0.72 \%$, respectively $(\mathrm{P}<0.001)$.

Knockdown of Aurora A reduced cell proliferation in vivo. Ki-67 staining was performed to detect cell proliferation. As shown in Fig. 5B, within a similar field of view, fewer proliferation cells (with brown staining) in tumor tissues were observed in the mice treated by pGenesil-2-shAURKA plasmid than glucose and pGenesil-2-HK treatment groups. The Ki67-labeling index was significantly smaller in pGenesil-2shAURKA treated group than glucose and pGenesil-2-HK treatment groups with values of $21.94 \pm 2.41 \%$ vs. $39.59 \pm$ $5.06 \%$ and $34.76 \pm 2.46 \%$, respectively $(\mathrm{P}=0.002)$.

\section{Discussion}

In the current study, we used the vector-based shRNA against Aurora A expression drived by U6 promoter to study the effect on human breast cancer cells both in vitro and in vivo. Our results showed that pGenesil-2-shAURKA plasmid could specifically knockdown the expression of Aurora A in vitro and in vivo, and inhibit tumor growth by systematic administration in animal experiments.

Aurora A protein is also known as serine threonine kinase 15 (STK15), BTAK, Aurora kinase A, Aurora-2 or AIKI. This gene is a member of the Aurora kinase family made up of Aurora A, B and C, and is conserved throughout eukaryotic 
A
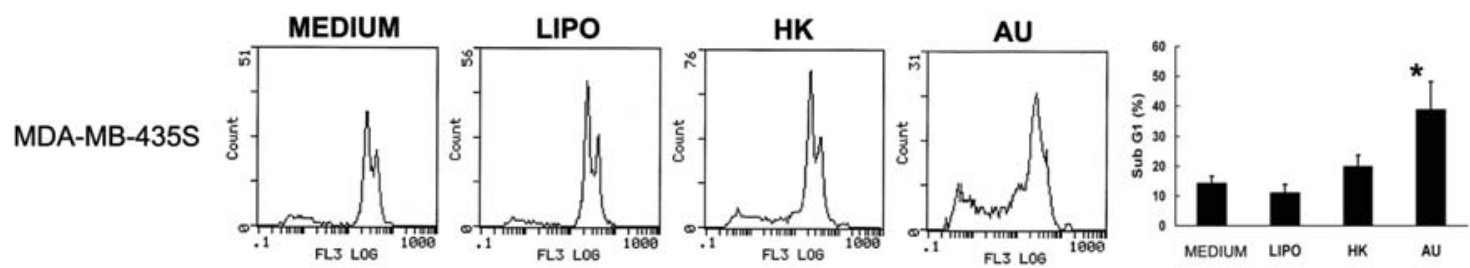

ZR-75-30

B
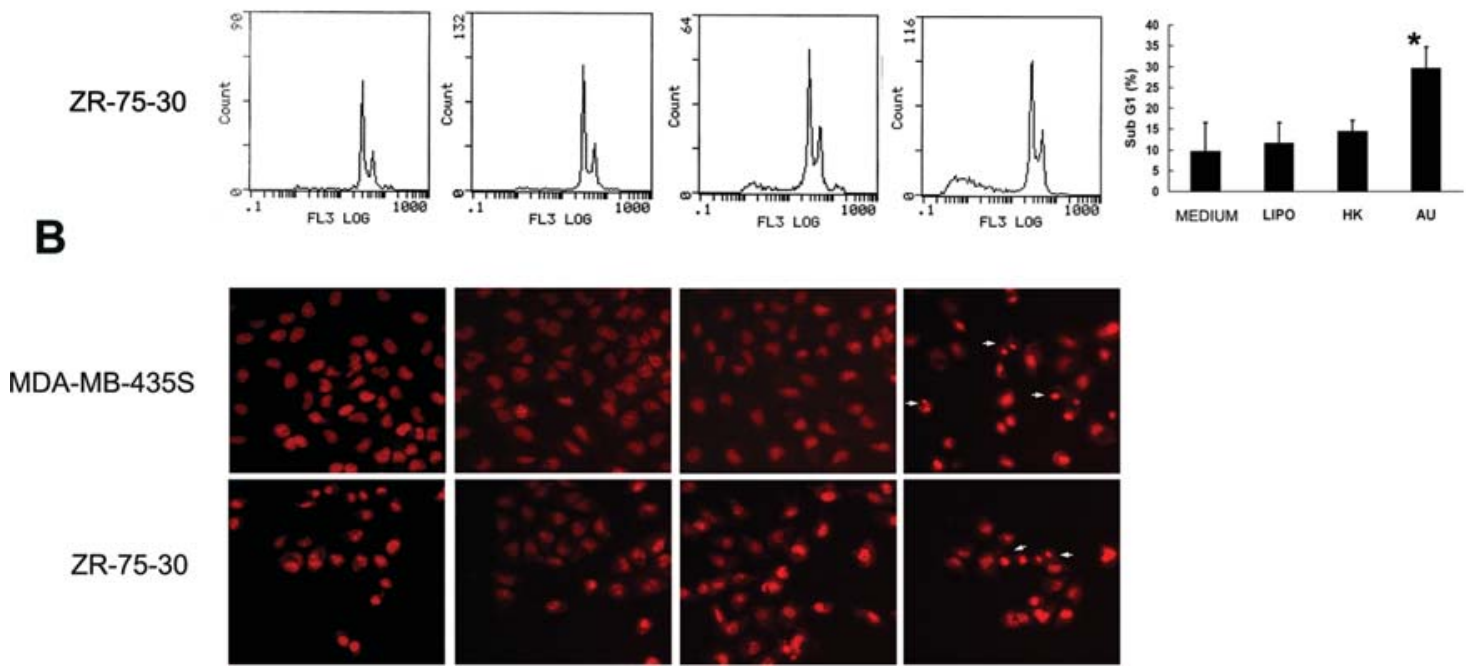

Figure 2. pGenesil-2-shAURKA induces apoptosis in vitro. (A) Cells were collected $48 \mathrm{~h}$ after transfection and subsequently assayed for their DNA content by flow cytometry. These experiments were performed 3 times. The average sub-G1 population were shown. ${ }^{*} \mathrm{P}<0.05$ compared with MEDIUM group. (B) Cells were transfected with pGenesil-2-shAURKA (AU) or pGenesil-2-shHK (HK)/liposome complex or liposome (LIPO) or medium (MEDIUM) alone as control for $48 \mathrm{~h}$, stained with PI, and examined under a fluorescence microscope (x200). These experiments were performed 3 times, and presentative results are shown. White arrows indicate examples of apoptotic cells.

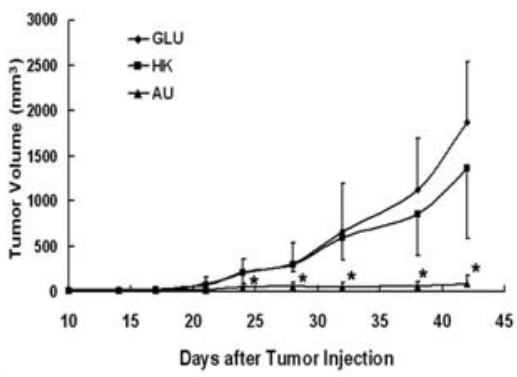

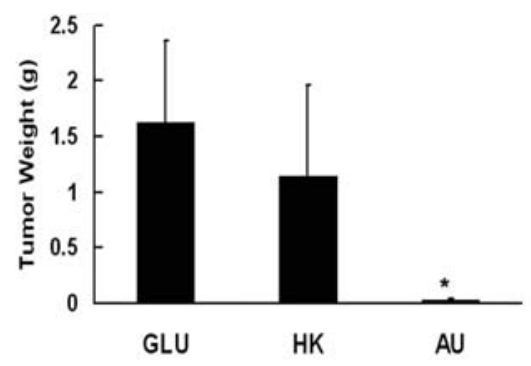

C
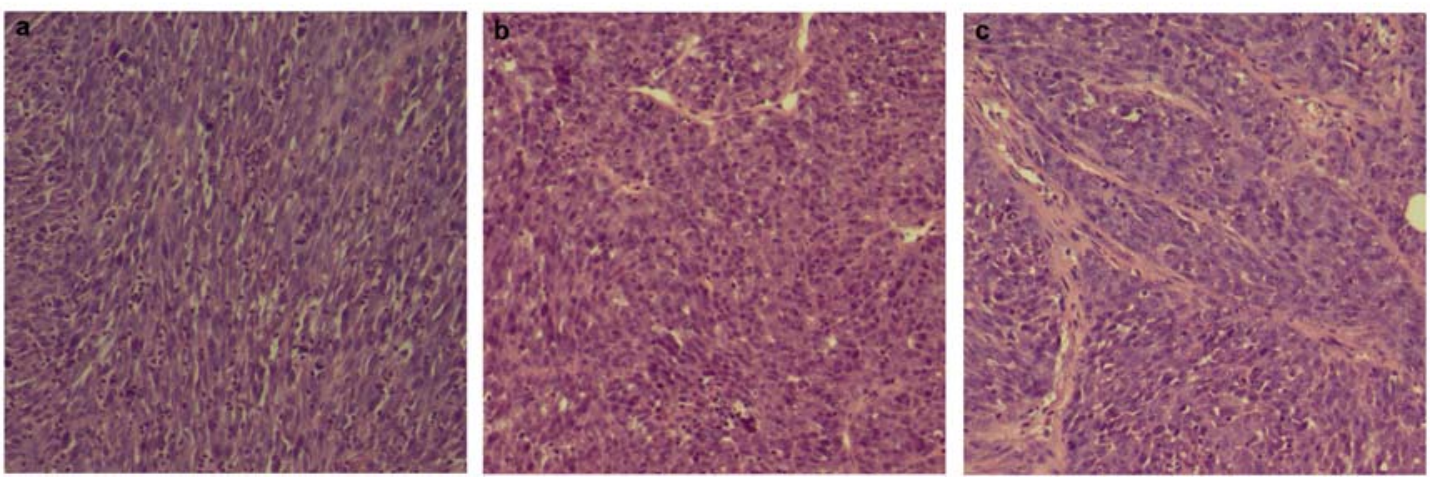

Figure 3. pGenesil-2-shAURKA inhibits tumor growth in MDA-MB-435S xenogenic implantation model. Female nude mice at 6-8 weeks of age were implanted subcutaneously with MDA-MB-435S cells. Ten days after tumor cells were implanted, the mice were assigned randomly to three groups and treated with pGenesil-2-shAURKA (AU) or pGenesil-2-shHK (HK)/liposome complex or 5\% glucose alone (GLU). (A) Suppression of tumor growth in mice. The sizes $\left(\mathrm{mm}^{3}\right)$ of tumors were monitored and recorded. Values are means $\pm \mathrm{SD}$. $\mathrm{N}=5,{ }^{*} \mathrm{P}<0.05$ compared with GLU group. (B) Three days after the last treatment, mice were sacrificed and subcutaneous tumors were weighed. $\mathrm{N}=5$, ${ }^{\text {"P }}<0.05$; compared with GLU group. (C) H\&E staining of subcutaneous tumors treated with glucose (a), pGenesil-2-shHK (b), and pGenesil-2-shAURKA (c) are shown. (Original magnification, x200). 
A GLU HK AU

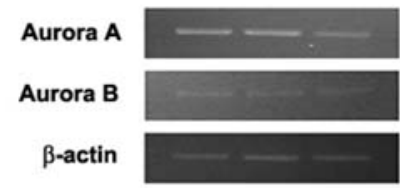

B

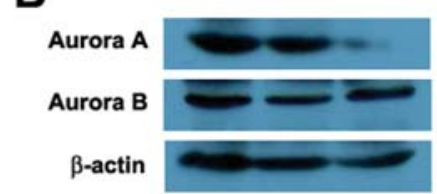

D

C
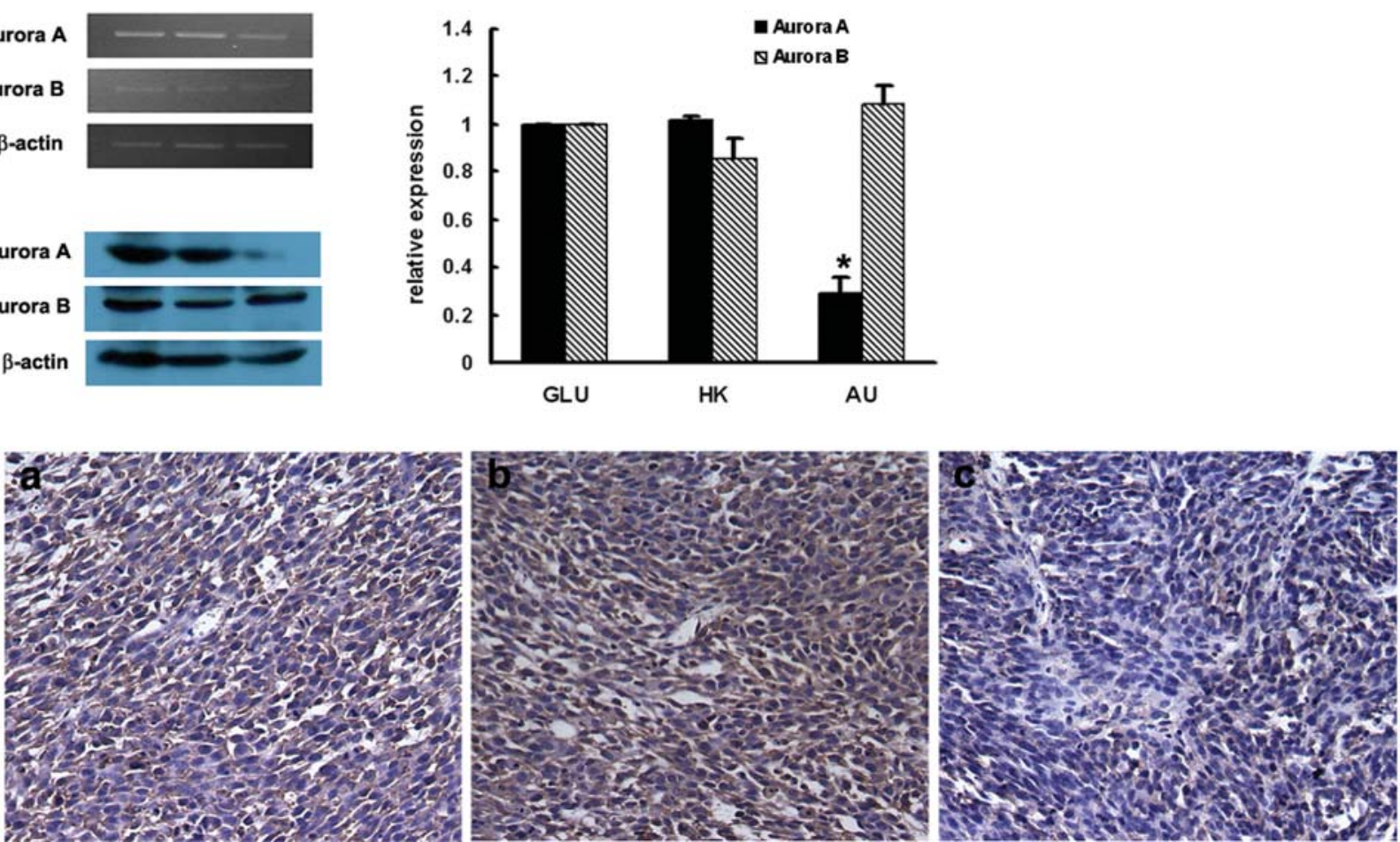

Figure 4. pGenesil-2-shAURKA specifically inhibits Aurora A expression in vivo. Three days after the last treatment, mice were sacrificed and the tumor tissues of each group were collected to study the expression of Aurora A and B. (A) Expression of Aurora A and B were detected by RT-PCR. (B) Expression of Aurora A and B were detected by Western blot, B-actin expression was monitored as the control. (C) The ratio of Aurora A (or Aurora B)/ $\beta$-actin was calculated by using densitometry, and values were normalized by dividing by the ratio at GLU group. The average ratio of Aurora A (or Aurora B)/ 3 -actin are shown. Values are means $\pm \mathrm{SD}$. $\mathrm{N}=5,{ }^{*} \mathrm{P}<0.05$ compared with GLU group. (D) Tumor tissues from tumor-bearing mice treated with pGenesil-2-shAURKA (c) or pGenesil-2-shHK (b) liposome complex or $5 \%$ glucose (a) were immunostained with Aurora A (original magnification, x200).

A

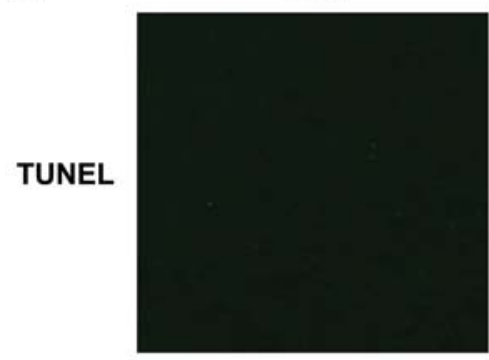

B

Ki-67
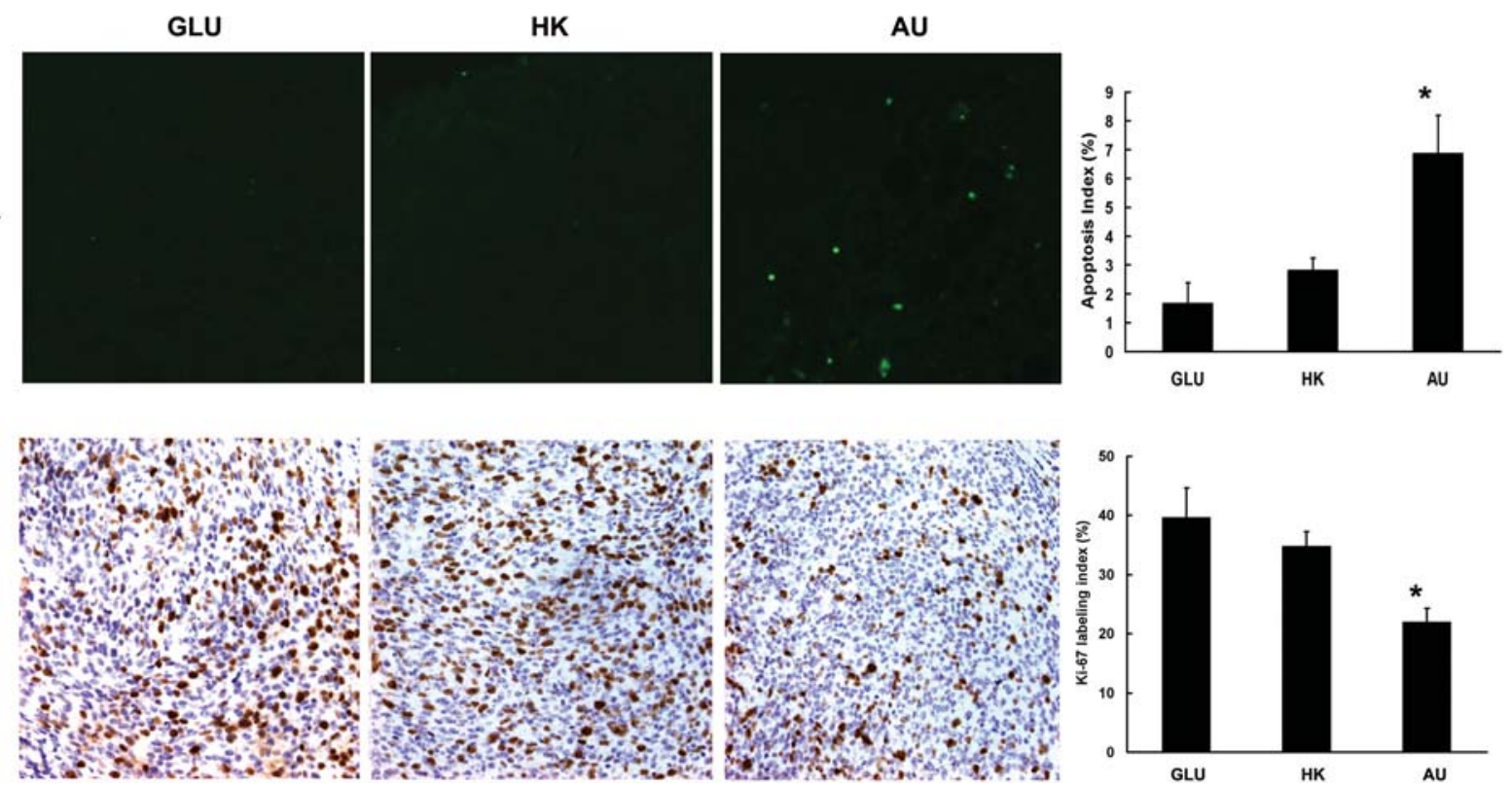

Figure 5. pGenesil-2-shAURKA induces apoptosis and inhibits cell proliferation in vivo. (A) Tumor tissues from tumor-bearing mice treated with pGenesil-2shAURKA (AU) or pGenesil-2-shHK (HK)/liposome complex or 5\% glucose (GLU) alone were sectioned and stained with FITC-dUTP, apoptotic cells (green) were identified and examined under a fluorescence microscope (original magnification, $\mathrm{x} 200$ ). The apoptotic index was calculated as a ratio of the apoptotic cell number to the total cell number in each field. Values are means $\pm \mathrm{SD}$. N $=5,{ }^{*} \mathrm{P}<0.05$ compared with GLU group. (B) Tumor tissues were sectioned and stained with Ki-67. Ki-67-labeling index was estimated as the percentage of neoplastic cells with positive nuclear staining of the total number of neoplastic cells counted. Values are means $\pm \mathrm{SD} . \mathrm{N}=5,{ }^{*} \mathrm{P}<0.05$ compared with GLU group (original magnification, $\mathrm{x} 200$ ). 
evolution. Aurora A regulates the correct development of the various phases of mitosis, including centrosome maturation and separation, mitotic entry, bipolar spindle assembly, chromosome alignment on the metaphase plate and cytokinesis (20). By the G2 phase of the cell cycle through anaphase, it can be detected in the pericentriolar material. Additionally, it spreads to mitotic spindle poles and midzone microtubules during metaphase (21). Hirota et al reported that following activation by the LIM protein ajuba in G2, initial activation of Aurora A in late G2 phase of the cell cycle is essential for recruitment of the cyclin $\mathrm{B} 1-\mathrm{Cdk} 1$ complex to centrosomes, it becomes activated and commits cells to mitosis (22). Aurora A interacts with TPX2, and it plays a role in the spindle assembly process (23). Katayama et al demonstrated that Aurora A played key roles at two steps in kinetochore/ chromatin-associated microtubule formation process. These include formation of $\alpha$-tubulin foci in the vicinity of the kinetochore/chromatin for microtubule nucleation and stabilization of the microtubules through feedback regulatory interactions with INCENP and TPX2 proteins (24).

In previous studies, the overexpression of Aurora A or amplication of chromosome 20q13 amplification, where human Aurora A is located at, has been detected in primary breast tumor samples and breast cancer cell lines (12). Moreover, in an MMTV-Aurora-A transgenic mouse model, enhanced branch morphogenesis in the mammary gland and more developed mammary tumors were detected at 20 months of age, which established Aurora A as an oncogene (25). Nadler et al reported that Aurora A expression defined a population of patients with decreased survival, suggesting that Aurora A might be the preferred drug target in breast cancer (13). Lee et al reported that treatment of MCF-7 breast cancer cells with 173 -estradiol (E2) in short-term resulted in the upregulation of Aurora A levels, and downregulation of Aurora A by RNA interference led to a significant decrease in estrogen-induced, anchoragedependent and independent growth of MCF-7 cells. Moreover, the knockdown of Aurora A could overcome estrogen-induced decrease in docetaxel sensitivity of MCF-7 cells. Above studies provide a clue that inhibiting the activity or knocking down the expression of Aurora A may be a new way to treat breast cancer (26). In the current study, we recombined an shRNA targeted on Aurora A into a plasmid vector, and used this construct as a specific inhibitor against Aurora A.

Aurora kinase inhibitors have shown anticancer effects both in vitro and in vivo, including ZM447439, Hesperadin 8 and VX-680, which have already undergone preclinical or phase I/II clinical trials. However, because most of the small molecules with Aurora A inhibitor function identified so far have been shown good specificity for the ATP-binding site, it may well be that the anticancer action of some chemotherapy agents do not reflect the blocking activity on Aurora A but on other family members (mainly Aurora B) or protein kinase. For instance, ZM447439 inhibits Aurora A and B, and VX-680 inhibits all three (27). Currently, several research groups, including our laboratory, have been involved in the development of specific Aurora A inhibitors (28,29; Jones et al, Proc ASCO 25: abs. 3577, 2007). In the present study, we utilized vector-based shRNA technique and constructed the recombi- nant plasmid expressing AURKA-shRNA to knock down the expression of Aurora A in vitro and in vivo. Based on the results of RT-PCR and Western blot, we confirmed that the specific AURKA-shRNA designed and used in this study successfully reduced the expression of Aurora A, and had little effect on the expression of Aurora B, indicating that vector-based shRNA may be used as an effective specific inhibitor of Aurora A.

The utilization of siRNA to knock down expression of a specific gene has become a method of choice for cell culture or in vitro studies. However, it is limited primarily by the cost of synthetic oligonucleotides and the need for repeated chemical synthesis. Increasing effort has been made to develop methods to deliver an expression cassette to produce the RNAi intermediate $(17,30,31)$, including shRNA (32). Moreover, unintended effects on gene expression mediated by RNAi termed 'off-target effects' are issues to take into consideration. Compared with chemically synthesized siRNA, shRNA is less likely to induce specific and non-specific offtarget effects, because shRNA is spliced by endogenous mechanisms (36). Besides, recent works suggested that, among vector-based RNAi systems, the shRNA system is more effective than the tandem system or siRNA is (33-35). Our results showed that, by systematic administration, pGenesil-2-shAURKA inhibited tumor growth in MDA-MB435S xenogenic implantation model obviously after specific knocking down of Aurora A expression. In current study, we did not find any side-effect in animals. In addition, pGenesil-2 vector used in this study contains the kanamycin resistance gene for selection in $E$. coli, thus ampicillin which may cause an allergic response in some individuals can be avoided. Our results suggested that pGenesil-2-shAURKA plasmid may be a potential therapy for human breast cancer.

Moreover, to further investigate the underlying mechanisms, we evaluated the effect of pGenesil-2-shAURKA on cell apoptosis and proliferation. In pacreatic cancer, colorectal cancer, non-small cell lung cancer and multiple myeloma cell lines, specific suppression of expression of Aurora A lead to G2-M arrest and apoptosis, and can enhance chemosensitivity and radiation response $(18,37,38)$. It is reported that Aurora A activates Akt-induced cell survival and chemoresistance in a p53-dependent manner in ovarian cancer cells (39). Wang et al reported that overexpression of Aurora A kinase promotes tumor cell proliferation and inhibits apoptosis in esophageal squamous cell carcinoma cell line (40). Besides, associations of overexpression of Aurora A and cell proliferation were detected in glioblastoma patients (41). In our study, by FCM, PI staining, TUNEL and Ki-67 immunostaining, increased apoptosis and reduced cell proliferation were detected after pGenesil-2-shAURKA treatment. Our results suggested increased apoptosis and reduced proliferation may be the mechanism of antitumor effect of pGenesil-2-shAURKA.

In summary, the current study suggested that vector based AURKA-shRNA may be a potential therapy for human breast cancer. Although the biodistribution and tumor accumulation of plasmid/liposome complex were investigated intensively $(42,43)$, the detailed biodistribution of systematic administration of pGenesil-2-shAURKA/liposome complex needs to be further investigated. Moreover, based on research 
on the relationship between Aurora A and radiation and chemotherapy of cancer cells $(18,25,26)$, the effect of AURKA-shRNA combined with radiation or chemotherapy might be a future direction.

\section{Acknowledgments}

This work is supported by Hi-tech Research and Development Program (863 Program) of China (2007AA021008 and 2009ZX09102-241). The National Key Basic Research Program (973 Program) of China (2010CB529900).

\section{References}

1. Parkin DM, Bray F, Ferlay $\mathrm{J}$ and Pisani P: Global cancer statistics, 2002. CA Cancer J Clin 55: 74-108, 2005.

2. Porter P: 'Westernizing' women's risks? Breast cancer in lowerincome countries. N Engl J Med 358: 213-216, 2008.

3. Joanne L: Breast cancer in 2007 incidence risk assessment and risk reduction strategies. Clin J Oncol Nurs 11: 619-622, 2007.

4. De Laurentiis M, Cancello G, D'Agostino D, et al: Taxanebased combinations as adjuvant chemotherapy of early breast cancer: a meta-analysis of randomized trials. J Clin Oncol 26: $44-53,2008$

5. Sparano JA, Wang M, Martino S, et al: Weekly paclitaxel in the adjuvant treatment of breast cancer. N Engl J Med 358: 1663-1671, 2008 .

6. Warner SL, Bearss DJ, Han H and Von Hoff DD: Targeting Aurora-2 kinase in cancer. Mol Cancer Ther 2: 589-595, 2003.

7. Kimura M, Kotani S, Hattori T, et al: Cell cycle-dependent expression and spindle pole localization of a novel human protein kinase, Aik, related to Aurora of Drosphila and yeast Ipl1. J Biol Chem 272: 13766-13771, 1997.

8. Berdnik D and Knoblich JA: Drosophila Aurora-A is required for centrosome maturation and actin-dependent asymmetric protein localization during mitosis. Curr Biol 12: 640-647, 2002.

9. Giet R, McLean D, Descamps S, Lee MJ, Raff JW, Prigent C and Glover DM: Drosophila Aurora A kinase is required to localize D-TACC to centrosomes and to regulate astral microtubules. J Cell Biol 156: 437-451, 2002.

10. Hannak E, Kirkham M, Hyman AA and Oegema K: Aurora-A kinase is required for centrosome maturation in Caenorhabditis elegans. J Cell Biol 155: 1109-1116, 2001.

11. Bischoff JR, Anderson L, Zhu Y, et al: A homologue of Drosophila aurora kinase is oncogenic and amplified in human colorectal cancers. EMBO J 17: 3052-3065, 1998.

12. Montembault E and Prigent C: Aurora kinases: therapeutic potential. Drugs Future 30: 29, 2005

13. Nadler Y, Camp RL, Schwartz C, Rimm DL, Kluger HM and Kluger Y: Expression of Aurora A (but not Aurora B) is predictive of survival in breast cancer. Clin Cancer Res 14: 4455-4462, 2008.

14. Mario Stevenson: Therapeutic potential of RNA interference. N Engl J Med 351: 1772-1777, 2004

15. Boudreau RL and Davidson BL: RNAi therapy for neurodegenerative diseases (Review). Curr Top Dev Biol 75: 73-92, 2006.

16. Campochiaro PA: Potential applications for RNAi to probe pathogenesis and develop new treatments for ocular disorders. Gene Ther 13: 559-562, 2006.

17. Lee NS, Dohjima T, Bauer G, et al: Expression of small interfering RNAs targeted against HIV-1 rev transcripts in human cells. Nat Biotechnol 20, 500-505, 2002.

18. Hata T, Furukawa T, Sunamura M, et al: RNA interference targeting Aurora kinase A suppresses tumor growth and enhances the taxane chemosensitivity in human pancreatic cancer cells. Cancer Res 65: 2899-2905, 2005.

19. Yuan Z, Liu H, Yan F, et al: Improved therapeutic efficacy against murine carcinoma by combining honokiol with gene therapy of PNAS-4, a novel pro-apoptotic gene. Cancer Sci 100: 1757-1766, 2009.

20. Marumoto T, Zhang D and Saya H: Aurora-A - a guardian of poles. Nat Rev Cancer 5: 42-50, 2005.

21. Fu J, Bian M, Jiang Q and Zhang C: Roles of aurora kinases in mitosis and tumorigenesis. Mol Cancer Res 5: 1-10, 2007.
22. Hirota T, Kunitoku N, Sasayama T, et al: Aurora-A and an interacting activator, the LIM protein Ajuba, are required for mitotic commitment in human cells. Cell 114: 585-598, 2003.

23. Kufer TA, Silljé HH, Körner R, Gruss OJ, Meraldi P and Nigg EA: Human TPX2 is required for targeting Aurora-A kinase to the spindle. J Cell Biol 158: 617-623, 2002.

24. Katayama H, Sasai K, Kloc M, Brinkley BR and Sen S: Aurora kinase-A regulates kinetochore/chromatin associated microtubule assembly in human cells. Cell Cycle 7: 2691-2704, 2008.

25. Wang X, Zhou YX, Qiao W, Tominaga Y, Ouchi M, Ouchi T and Deng CX: Overexpression of aurora kinase A in mouse mammary epithelium induces genetic instability preceding mammary tumor formation. Oncogene 25: 7148-7158, 2006.

26. Lee HH, Zhu Y, Govindasamy KM and Gopalan G: Downregulation of Aurora-A overrides estrogen-mediated growth and chemoresistance in breast cancer cells. Endocr Relat Cancer 15: 765-775, 2008.

27. Agnese V, Bazan V, Fiorentino FP, et al: The role of Aurora-A inhibitors in cancer therapy. Ann Oncol 18: vi47-vi52, 2007.

28. Coumar MS, Leou JS, Shukla P, et al: Structure-based drug design of novel Aurora kinase A inhibitors: structural basis for potency and specificity. J Med Chem 52: 1050-1062, 2009.

29. Deng XQ, Wang HY, Zhao YL, et al: Development and biological evaluation of a novel Aurora A kinase inhibitor pharmacophore modelling and virtual screening for identification of new Aurora-A kinase inhibitors. Chem Biol Drug Des 71: 533-539, 2008.

30. Miyagishi $\mathrm{M}$ and Taira K: U6 promoter-driven siRNAs with four uridine $3^{\prime}$ overhangs efficiently suppress targeted gene expression in mammalian cells. Nat Biotechnol 20: 497-500, 2002.

31. Paul CP, Good PD, Winer I and Engelke DR: Effective expression of small interfering RNA in human cells. Nat Biotechnol 20: 505-508, 2002.

32. Leung RK and Whittaker PA: RNA interference: from gene silencing to gene-specific therapeutics. Pharmacol Ther 107: $222-239,2005$.

33. Miyagishi $\mathrm{M}$ and Taira $\mathrm{K}$ : Strategies for generation of an SiRNA expression library directed against the human genome. Oligonucleotides 13: 325-333, 2003.

34. Siolas D, Lerner C, Burchard J, et al: Synthetic shRNAs as potent RNAi triggers. Nat Biotechnol 23: 227-231, 2005.

35. Wu MT, Wu RH, Hung CF, Cheng TL, Tsai WH and Chang WT: Simple and efficient DNA vector-based RNAi systems in mammalian cells. Biochem Biophys Res Commun 330: 53-59, 2005.

36. Rao DD, Vorhies JS, Senzer N and Nemunaitis J: siRNA vs. shRNA: similarities and differences. Adv Drug Deliv Rev 61: 746-759, 2009.

37. Tao Y, Zhang P, Frascogna V, Lecluse Y, Auperin A, Bourhis J and Deutsch E: Enhancement of radiation response by inhibition of Aurora-A kinase using siRNA or a selective Aurora kinase inhibitor PHA680632 in p53-deficient cancer cells. Br J Cancer 97: 1664-72, 2007

38. Evans R, Naber C, Steffler T, et al: Aurora A kinase RNAi and small molecule inhibition of Aurora kinases with VE-465 induce apoptotic death in multiple myeloma cells. Leuk Lymphoma 49: 559-569, 2008

39. Yang H, He L, Kruk P, Nicosia SV and Cheng JQ: Aurora-A induces cell survival and chemoresistance by activation of Akt through a p53-dependent manner in ovarian cancer cells. Int J Cancer 119: 2304-2312, 2006.

40. Wang XX, Liu R, Jin SQ, Fan FY and Zhan QM: Overexpression of Aurora-A kinase promotes tumor cell proliferation and inhibits apoptosis in esophageal squamous cell carcinoma cell line. Cell Res 16: 356-366, 2006.

41. Samaras V, Stamatelli A, Samaras E, et al: Comparative immunohistochemical analysis of aurora-A and aurora-B expression in human glioblastomas. Associations with proliferative activity and clinicopathological features. Pathol Res Pract 205: 765-773, 2009.

42. Mahato RI, Anwer K, Tagliaferri F, et al: Biodistribution and gene expression of lipid/plasmid complexes after systemic administration. Hum Gene Ther 9: 2083-2099, 1998.

43. Zhang Y, Bradshaw-Pierce EL, Delille A, Gustafson DL and Anchordoquy TJ: In vivo comparative study of lipid/DNA complexes with different in vitro serum stability: Effects on biodistribution and tumor accumulation. J Pharm Sci 97: 237$250,2008$. 